\title{
Comparison of two self-help smoking cessation booklets
}

\author{
Kevin P Balanda, John B Lowe, Mary-Louise O'Connor-Fleming
}

\begin{abstract}
Objective-To compare two self-help smoking cessation booklets distributed to callers to a Quitline telephone service in Queensland (Australia).

Design-Callers were randomised to receive either a structured 14-day quit programme (Time to quit) or another booklet that described four broad stages of quitting (Can quit). Approximately one month later, these callers were interviewed by telephone.

Main outcome measures-Self-reported smoking status at one month and recent quit attempts together with process measures.

Results-Altogether, 521 callers (78.3\%) were interviewed. They were heavier smokers when compared with all Queensland smokers: on average they had smoked for more than 15 years, smoked nearly 25 cigarettes per day, and almost two-thirds had attempted to quit smoking in the past year. In each group, significant proportions either did not begin to use the booklet $(50.5-56.0 \%)$, or did not complete its use $(77.4-82.3 \%)$. There were no differences in the self-reported quit rates at one month $(17.0 \%$ vs $16.1 \%$; $p=0.93)$. In an ordinal regression modelling procedure involving age, sex, number of recent quit attempts, number of cigarettes smoked per day, smoking status of partner, number of five closest friends who smoke, education, and booklet received, only the number of cigarettes smoked per day was significantly related to smoking status at one month.
\end{abstract}

Conclusions-Callers to telephone Quitline services are typically heavier smokers than the general smoking population, and simple strategies, such as self-help booklets, appear to achieve relatively high success. Nevertheless, there is potential to improve the effectiveness of these materials by making a range of materials available and encouraging callers to make a serious attempt to quit smoking.

(Tobacco Control 1999;8:57-61)

Keywords: smoking cessation, self-help materials, telephone quitlines

\section{Introduction}

Studies have consistently shown that smokers who achieve long-term cessation significantly reduce their risk of disability or early death, and lower the healthcare costs to the community. ${ }^{1}$ Tobacco smoking is the largest single preventable cause of death in Australia. In 1992, English et al ${ }^{2}$ estimated that smoking caused 18920 deaths in Australia; this is nine times the number of road crash fatalities. In the same year, premature tobacco-related deaths before the age of 70 claimed 88266 person-years of life, and led to 98373 hospital episodes and the use of 812866 hospital bed days. $^{2}$

Most smokers state that they would attempt cessation if effective and acceptable means of assistance were made available to them. ${ }^{3}$ Studies have examined smokers' preferences for different forms of assistance with cessation, and variables that may be associated with these preferences. ${ }^{4}$ These variables included smoking rate, confidence about likelihood of success with cessation, perception of difficulty, number of previous cessation attempts, duration of previous periods of abstinence, and reasons for previous relapses. Smokers' preferences for strategies that assisted with smoking cessation included: help from a medical practitioner; help from another health professional; using a book, a pamphlet or a quit kit; a stop-smoking group; and a television programme or video. ${ }^{4} \mathrm{~A}$ small number of smokers preferred help in the form of a lecture, by telephone, or by mail. ${ }^{4}$

Owen $e t a l^{3}$ examined the characteristics of 613 smokers who called a telephone counselling service and conducted a 12-month follow up of a randomly selected sample of callers, half of whom had been given further telephone advice or other cessation materials three months after their initial call. Results suggested that callers were more likely to be addicted, to be heavy-smokers, and to have smoked the first cigarette of the day within the first 30 minutes of waking. They were least likely to be able to quit without assistance, and more likely to be women and to be younger.

Printed self-help materials can be effective in helping smokers to achieve and maintain smoking abstinence. ${ }^{3}$ Evidence suggests that the most appropriate content and design of such materials includes information about the health and social consequences of smoking, specific strategies and exercises for quitting, and specific strategies and exercises for non-smoking, avoidance of relapse, and recycling in case of initial failure or relapse. ${ }^{5}$ Further, Orleans et $a l^{6}$ found that, in a trial of self-help materials, results suggested that naturally occurring social support for quitting strongly predicted long-term cessation. Minimal levels of social support or therapist contact in person or by telephone are also reported to enhance the effects of self-help 
materials. ${ }^{7}$ Higher levels of reported adherence to the content of self-instructional materials may be associated with a greater likelihood of cessation. ${ }^{6} 7$

Self-help, minimal-intervention strategies for smoking cessation may be more easily assessed, reducing barriers to participation by smokers. ${ }^{58}$ Although these programmes produce lower quit rates $(20 \%)$ at 12 -month follow up ${ }^{7}$ compared with more intense programmes, the total number of smokers who request the materials and participate in the programme, if higher than intensive programmes, will produce a greater number of non-smokers.

During the past decade, self-help materials have been developed using Prochaska and DiClemente's model of stages of change. ${ }^{9}$ Programmes based on this model that have been used in general practice have been demonstrated to be effective. ${ }^{10}$ In addition, programmes based on a structured activity format giving the smoker a day-by-day process to quit, are also still available. Currently, a telephone service is available in Australia for smokers wishing to quit. This national service is run by each state independently. By calling one number the smoker is directed to the service in their own state. In Queensland, the Quitline service can select from a variety of materials to provide to the smoker.

This study compared the effectiveness of two different styles of self-help cessation booklets distributed to callers to a Quitline telephone service in Queensland. The principal research question was to determine if one booklet should be recommended over another for distribution to callers to the Quitline.

\section{Methods}

MATERIALS

The first booklet (Can quit) was a colourful publication that emphasised four broad stages of quitting (Deciding to quit, Preparing to quit, Quitting, Staying a non-smoker) and contained colourful photographic images. This booklet is available nationally. It allows the reader to pick and choose sections of the materials that are most relevant. The programme is based on the Prochaska and DiClemente stages-of-change model. Information is available for smokers at different stages in their quitting. It does not guide the reader through activities in a step-by-step fashion. The second booklet (Time to quit) contained a structured 14-day quitting programme without colourful photographs. This booklet is available locally and distributed by the state health department. The structured format does not make it easily available to select activities that are of relevance to the smoker. It does provide the smoker with a number of activities that the smoker may not have been aware of that might help them to quit. In contrast, it assumes smokers are in the action phase of the stages-of-change model. The availability of two different booklets was not promoted in a media campaign which simply encouraged smokers to call Quitline where a booklet could be requested without charge.
RECRUITMENT

Seven hundred and eight consecutive callers to Quitline were recruited into the study. During the call to Quitline, no systematic smoking cessation counselling was given. Callers were randomised into two groups: members of the first group were sent the Time to quit booklet, whereas those in the second group were sent the Can quit booklet. The Time to quit booklet group is currently used by Quitline and approximately twice as many callers were randomised to receive it. Callers were asked to give verbal consent to be contacted later, and their telephone number and details of the best time to call were recorded. When the caller was calling on behalf of some other smoker, contact details of that smoker were recorded.

\section{QUITLINE INFORMATION}

During a Quitline call, details were collected about the age, sex, and postcode of residence of the caller, and their relationship to the smoker. In addition, the following details were collected about the smoker: how long they had smoked, if they were currently smoking, the average number of cigarettes smoked per day, when they had their first cigarette after waking, and number of recent quit attempts.

\section{ONE-MONTH TELEPHONE INTERVIEWS}

One-month interviews were conducted by experienced telephone interviewers using a computer assisted telephone interviewing (CATI) system. Interviewers underwent a thorough briefing session before the survey and all fieldwork was monitored by the CATI supervisor who audited a sample of interviews to verify the accuracy of recorded responses. Initial calls were made between $5 \mathrm{pm}$ and $9 \mathrm{pm}$ on weekday evenings. Up to four callbacks were made before a Quitline caller was registered as "non-contactable." When no specific time was obtained, callbacks were made later during that evening, on another weekday evening, on the following Saturday, and finally during a weekday.

The questionnaire gathered opinions about the amount of information in the booklets and the extent to which they were used. Self-reported smoking cessation details were also collected: self-ascribed current smoking status (current smoker/ex-smoker) and time since last puff of a cigarette (within the past two days, more than two days ago but less than seven days, more than seven days ago). Details of self-reported quit attempts in the intervening period included whether or not they had been without a cigarette for at least 24 hours, the most number of days without a cigarette, and whether or not they had resumed smoking. Details about the smoking status of their partner, the number of their five closest friends who smoked, and the smoker's highest level of education (primary, secondary, tertiary) were also gathered.

Pilot testing of the telephone questionnaire was undertaken among 25 smokers who called Quitline before the study. They were sent a copy of the draft questionnaire and interviewed over the phone. This resulted in a number of 
changes to the questionnaire and improved its face and content validity.

ANALYSIS

$\chi^{2}$ statistics, Fisher's exact test for $2 \times 2$ tables, and independent Student's $t$ tests were used to assess possible response bias in the one-month telephone survey and group differences in responses. An ordinal regression analysis using variable selection procedures, including all the above variables as independent variables, was then used to explore factors relating to self-reported smoking cessation at one month. All statistical analyses were done using the statistical package SAS.

\section{Results}

Seven hundred and eight initial callers to Quitline agreed to participate in the study. Most of the callers were the smokers themselves (table 1). During the one-month telephone survey, 43 were found to be ineligible for various reasons. Examples of these ineligible callers included persons who had called the Quitline on behalf of a smoker and the smoker did not wish to quit, whereas others had called requesting booklets for student school projects. Eighty of the remaining 665 eligible callers could not be contacted and 64 of the eligible subjects who was contacted refused to be interviewed. A total of 521 completed follow-up interviews was obtained. For six of these, no Quitline information was available and they were excluded from analyses using such information. This gave a contact rate of $88.0 \%$ (585/665), a consent rate of $89.1 \%(521 / 585)$, and an overall response rate of $78.3 \%$ (521/665). A comparison of the Quitline information of those callers who were subsequently interviewed and those who were not revealed two statistically significant differences. Respondents to the telephone survey were more likely to have called Quitline for themselves $(90.6 \%$ vs $82.5 \%, p=0.006)$ and to live outside Brisbane $(72.2 \%$ vs $63.4 \%$; $=0.047)$.

\section{ONE-MONTH TELEPHONE SURVEY}

Overall, $362(69.5 \%)$ respondents to the one-month telephone survey received the Time to quit booklet whereas the remaining 159 $(30.5 \%)$ received the Can quit booklet. Table 1 contains summary details about the sample of the 515 telephone respondents with Quitline information available. Over half were women, and nearly two-thirds were aged 35 years or over. As expected, nearly all were currently smoking and many could be described as heavy

Table 1 Quitline information on respondents to the one-month telephone survey, by group $(n=515)$

\begin{tabular}{llll}
\hline & $\begin{array}{c}\text { "Time } \\
\text { to quit" }\end{array}$ & $\begin{array}{l}\text { "Can } \\
\text { quit" }\end{array}$ & All respondents \\
\hline Called Quitline themselves $(\mathrm{p}=0.02)$ & $92.8 \%$ & $86.5 \%$ & $90.9 \%$ \\
Male $(\mathrm{p}=0.67)$ & $42.6 \%$ & $44.8 \%$ & $43.3 \%$ \\
Aged under 35 years $(\mathrm{p}=0.06)$ & $33.4 \%$ & $42.3 \%$ & $36.1 \%$ \\
Lives outside Brisbane $(\mathrm{p}=0.21)$ & $73.4 \%$ & $67.8 \%$ & $71.8 \%$ \\
Currently smoking $(\mathrm{p}=0.77)$ & $97.5 \%$ & $96.8 \%$ & $97.3 \%$ \\
Mean number of years as a smoker $(\mathrm{p}=0.24)$ & 15.7 & 14.3 & 15.3 \\
Mean number of cigarettes per day (p=0.31) & 24.2 & 22.7 & 23.7 \\
First cigarette within 30 min of waking $(\mathrm{p}=0.04)$ & $76.9 \%$ & $67.2 \%$ & $74.1 \%$ \\
Had attempted to quit in past year $(\mathrm{p}=0.97)$ & $66.9 \%$ & $66.7 \%$ & $66.8 \%$ \\
\hline
\end{tabular}

Table 2 Process measures on respondents to the one-month telephone survey, by group $(n=521)$

\begin{tabular}{lll}
\hline & $\begin{array}{c}\text { "Time } \\
\text { to quit" } \\
\%\end{array}$ & $\begin{array}{l}\text { "Can } \\
\text { quit" } \\
\%\end{array}$ \\
\hline $\begin{array}{lll}\text { Found Quitline helpful }(\mathrm{p}=0.12) \\
\text { Recalled name of booklet }(\mathrm{p}=0.07)\end{array}$ & 92.8 & 88.7 \\
Read most or all of the booklet $(\mathrm{p}=0.58)$ & 29.0 & 21.4 \\
Started to use the booklet $(\mathrm{p}=0.25)$ & 41.0 & 68.6 \\
Completed using the booklet $(\mathrm{p}=0.02)$ & 17.7 & 44.0 \\
Booklet had (p $=0.54):$ & 22.6 \\
$\quad$ not enough information & 13.9 & 10.7 \\
$\quad$ just right amount of information & 82.0 & 84.0 \\
too much information & 4.1 & 5.3 \\
Still have the booklet $(\mathrm{p}=0.87)$ & 92.2 & 91.8 \\
\hline
\end{tabular}

smokers. Two-thirds had attempted to quit in the past year. There were only few statistically significant differences in the Quitline information of the two groups. Recipients of the Time to quit booklet were more likely to have called Quitline themselves $(92.8 \%$ vs $86.5 \%, \mathrm{p}=$ 0.02 ) and more likely to have their first cigarette within 30 minutes of waking $(76.9 \%$ vs $67.2 \%, \mathrm{p}=0.04)$.

Table 2 presents process measures collected from the 521 respondents to the one-month telephone survey. Overall, nearly all found Quitline helpful ("somewhat helpful" or "extremely helpful") with no significant group differences. Recipients of the Can quit booklet, with its colourful format, were less likely to recall its name than recipients of the Time to quit booklet, although this was not statistically significant. Nearly three-quarters had read most of the booklet ("all of it" or "most of it"). Fewer than half $(49.5 \%$ in the Time to quit group, $44.0 \%$ in the Can quit group) reported that they started to use the booklet sent to them. Although approximately equal percentages in the two groups started to use the booklets, significantly more completed using the Can quit booklet than the Time to quit booklet. One in five $(20.3 \%)$ recipients of the Time to quit booklet who started using the 14-day plan reported that they did not like working through the day-by-day process. On the other hand, $23.3 \%$ of recipients of the Can quit booklet reported that they would prefer to work through a day-by-day plan. After one month, most respondents still had their booklets.

There is some weak evidence that recipients of the Can quit booklets were more likely to have gone without a cigarette for more than 24 hours since calling Quitline $(58.9 \%$ vs $67.1 \%$, $\mathrm{p}=0.08)$. Moreover, although not statistically significant $(p=0.21)$, the Can quit group also tended to have gone longer without a cigarette than the Time to quit group (table 3). Although there appeared to be this weak evidence of more active quit attempts in the Can quit group, these differences had vanished by the end of the month. Overall, $16.3 \%$ of respondents reported at the end of the month that they had not had a puff of a cigarette in the past seven days. There were no statistically significant group differences $(p=0.93)$. Similarly, there were no statistically significant group differences in the percentages of respondents who self-described themselves at one month as ex-smokers $(23.4 \%$ vs $25.2 \%$, $\mathrm{p}=0.67)$. 
Table 3 Self-reported smoking outcomes on respondents to the one-month telephone survey, by $\operatorname{group}(n=521)$

\begin{tabular}{|c|c|c|}
\hline & $\begin{array}{l}\text { "Time } \\
\text { to quit" }\end{array}$ & $\begin{array}{l}\text { "Can } \\
\text { quit" }\end{array}$ \\
\hline Have gone without a cigarette for more than 24 hours $(p=0.08)$ & $58.9 \%$ & $67.1 \%$ \\
\hline Longest number of days without a cigarette (mean) $(\mathrm{p}=0.21)$ & 8.8 & 10.8 \\
\hline \multicolumn{3}{|l|}{ Smoking status at one month $(\mathrm{p}=0.93)$} \\
\hline Puff in past two days & $79.0 \%$ & $78.6 \%$ \\
\hline Puff in past seven days, but not in past two days & $5.0 \%$ & $4.4 \%$ \\
\hline No puff in past seven days & $16.1 \%$ & $17.0 \%$ \\
\hline
\end{tabular}

Separate ordinal regression models were used to assess the relationship between the self-reported smoking status at one month (puff in past two days, no puff in past two days but in past seven, no puff in past seven days) and the eight independent variables: age, sex, number of recent quit attempts, number of cigarettes smoked per day, smoking status of partner, number of five closest friends who smoke, education, and the booklet received. In no cases, except the number of cigarettes smoked per day and age, was there any evidence of a bivariate relationship. Those who had smoked larger number of cigarettes per day were less likely to report that they were not smoking at one month $(\mathrm{p}=0.037)$, whereas younger callers were marginally more likely $(\mathrm{p}=0.052)$.

To explore the simultaneous effect of these variables, an ordinal regression model of the self-reported smoking status at one month was fitted using a backward selection procedure starting with the eight variables. In total, 68 records $(13 \%)$ were dropped from this analysis because of missing data. The final model contained only one variable - number of cigarettes smoked per day when Quitline was called. In particular, the booklet received did not appear in the final model and was in fact the first variable removed. This same final model arose when a forward selection procedure and a stepwise procedure was used.

\section{Discussion}

A disproportionate number of callers to the Quitline service were from outside Brisbane, suggesting there may be a need for people living outside metropolitan areas to have access to a range of regionalised quit-smoking services. Glynn et $a l^{5}$ reported on the importance of self-help/minimal-intervention strategies for smoking cessation that included broad programme delivery modes, involving a range of approaches, and conducted over time in a range of settings, to increase motivation, opportunity, and likelihood of quitting smoking,

Callers to the Quitline service in this study were heavier smokers compared with all Queensland smokers. Moreover, callers to Quitline consisted of proportionately more women than men compared with all Queensland smokers. Owen et $a l^{3}$ reported on the characteristics of smokers who called a telephone counselling service, suggesting that callers were more likely to be addicted, to be heavy smokers, and to have smoked the first cigarette of the day within 30 minutes of waking. Further, they were least likely to be able to quit without assistance, and more likely to be women and to be younger. Fiore et al ${ }^{11}$ reported that in the American smoking population, most cigarette smokers who seek help tend to be heavier smokers. This poses particular challenges for self-help/minimalintervention strategies if cessation programmes attract more addicted smokers.

Although research suggests that smoking cessation programmes using printed self-help materials have been associated with abstinence rates of up to $20 \%$ at 12 -month follow up, ${ }^{7}$ fewer than half of the participants in this study reported that they had started to use the booklet sent to them. There was a clear underuse of the material, and greater uptake and continuation of use of printed self-help materials may occur if the need to make a serious attempt to quit is emphasised. The efficacy of self-instructional materials may be improved if participant progress can be monitored and if specific personalised feedback can be given. ${ }^{7}$ Important elements of self-help/minimalintervention strategies have been reported to include motivating more smokers to stop, using a variety of techniques to increase motivation, and targeting the programme to smoking cessation stages. $^{57}$

There was no discernible difference between one-month self-reported quit rates for recipients of the Can quit booklets and recipients of the Time to quit booklets. This is despite the fact that the Can quit booklet was colourful and emphasised four broad stages of quitting. The Time to quit booklet, on the other hand, had no colourful photographs and contained a structured 14-day quitting programme. Some have suggested that higher levels of adherence to the content of self-instructional materials may be associated with a greater likelihood of cessation. ${ }^{6}$ However, Brown and Owen ${ }^{7}$ reviewed research on self-help cessation programmes and concluded that there may not be any beneficial effect from the adherence to specific content of self-help materials. The content and design of self-help/minimal intervention programmes has been identified as an important element of intervention strategies. While the Can quit group were more likely to have gone without a cigarette for more than a day when compared with the Time to quit group, there were no differences in the self-reported quit rates at one month.

When combined with the fact that fewer than half of the participants in this study reported that they had started to use the booklet (either one) sent to them, these quit rates must be interpreted cautiously; they may not represent the total percentages of quitters. This study only followed up smokers one month after they called the Quitline service. This short period was selected to measure immediate uptake and impact of mailed materials. Further changes in quit rates may have been observed if follow up had continued to 18-24 months later. Previous work has demonstrated differences emerging from a longer follow-up period of 18 months. ${ }^{11}$ It was interesting to note that most of the smokers $(70 \%)$ did read 
the booklet, yet only a small percentage (20\%) completed the activities in the booklet, indicating that most of the smokers were in the contemplation or preparatory phase and not in the action phase.

Issues such as monitoring participant progress, specific personalised feedback and targeting programmes to smoking cessation stages may improve intervention success. ${ }^{57}$ The use of different styles of booklets in this study, did not produce significant differences to recommend the use of one booklet over the other. In fact, the use of both booklets should be considered. The study found that the non-targeted distribution of both booklets resulted in a relatively high level of non-use among participants, suggesting the need for alternative strategies to motivate and encourage smokers to use these booklets.

The Queensland Cancer Fund, using a grant from the Queensland Health Promotion Council, contracted the Centre for Health Promotion and Cancer Prevention Research to conduct this study. The authors would like to thank Michelle Sheldrake for her contribution and Peter Baade for statistical programming assistance, the Epidemiology and Health Information Branch of Queensland Health for the use of their CATI system, and the members of the Quitline telephone service for their cooperation.
1 Zhu S-H, Rosbrook B, Anderson C, et al. The demographics of help-seeking for smoking cessation in California and the role of the California Smokers' Helpline. Tobacco Control 1995;4(suppl 1):S9-15.

2 English DR, Holman CDJ, Milne E, et al. The quantification of drug caused morbidity and mortality in Australia, 1995 edition. Canberra, ACT: Department of Services and Health, tion. Can.

3 Owen N, Roberts L, Wakefield M. The South Australian Quit Advisory Service: characteristics of callers and cessation outcomes. Brief reports. Health Promotion fournal of Australia 1995;5:49-50.

4 Owen N, Davies MJ. Smokers' preferences of assistance with cessation. Prev Med 1990;19:424-31.

5 Glynn TJ, Boyd GM, Grurnan JC. Essential elements of self-help/minimal intervention strategies for smoking cessation. Health Educ Q 1990;17:329-45.

6 Orleans CT, Schoenbach VJ, Wagner EH, et al. Self-help quit smoking interventions: effects of self-help materials, social support instructions, and telephone counseling. $\mathcal{F}$ Consult Clin Psychol 1991;59:439-48.

7 Brown SL, Owen N. Self-help smoking cessation materials. Aust F Public Health 1992;16:188-91.

8 Ockene JK. Are we pushing the limits of public health interventions for smoking cessation? Health Psychol 1992; 11:277-9.

9 Prochaska JO, DiClemente CC. Stages and processes of self-change in smoking: toward an integrative model of change. F Consult Clin Psychol 1983;5:390-5.

10 Richmond RL. A smoking cessation programme for use in general practice. Med F Aust 1985;142:190-4.

11 Fiore MC, Novotny TE, Pierce JP, et al. Methods used to quit smoking in the United States: do cessation programs help? $7 A M A$ 1990;263:2760-5.

\section{Madagascar}

There are two approaches to confronting leeches. One is to be blasé, and wear shorts and sandals. The leeches find you, but you can find them equally easily. That's the Malagasy approach. That, and a pouch of tobacco powder to sprinkle on the transgressors so they drop off. The alternative approach is to attempt a defense with long trousers, wool socks and boots.

Howell KK, editor. Madagascar. "California Wild” 1998 (Fall):10.

California Academy of Sciences. Submitted by fudith Mackay 